\title{
FUNCTIONAL CLASSES OF NEURONS IN PRIMARY AUDITORY CORTEX OF THE CAT DISTINGUISHED BY SENSITIVITY TO SOUND LOCATION $^{1}$
}

\author{
JOHN C. MIDDLEBROOKS* AND JOHN D. PETTIGREW ${ }^{* 2}$ \\ * Coleman Memorial Laboratory, University of California, HSE-863, San Francisco, California 94143 and $\ddagger$ Beckman Laboratories of \\ Behavioral Biology, California Institute of Technology, 216-76, Pasadena, California, 91125
}

\begin{abstract}
Could neurons in the primary auditory cortex (AI) encode the spatial location of sounds? Is location sensitivity a parameter of stimulus coding relevant to topographical organization in AI? We have mapped receptive fields of single units in $\mathrm{AI}$ of the cat by varying systematically the location of a tonal stimulus within a sound field free of acoustic obstructions and reflections. By orienting tangential electrode penetrations parallel to the mediolateral axes along which neurons are tuned to the same stimulus frequency, we have explored the possibility that this axis could be concerned with spatial features of sound stimuli.

Approximately half of the neurons encountered were selective for the location of sounds. The locationselective units could be divided into two discrete populations. Hemifield units responded only to sounds presented in the contralateral sound field, with receptive fields extending from a well defined medial border to beyond the contralateral pole of the sound field. Axial units had small, completely circumscribed receptive fields that were constant in location for all such units in each cortical hemisphere studied. The location of axial receptive fields coincided with the acoustical "axis" of the contralateral pinna which was defined by acoustical measurements of the directionality of the ear. Axial units were restricted to the rostral pole of AI where neurons are tuned for high frequencies, while hemifield units were located more caudally and were tuned for lower frequencies. The location-insensitive, omnidirectional, units were distributed across the entire length of AI sampled. Some of the properties of the measured receptive fields could be inferred by comparing the passive acoustical properties of the ear with binaural interactions previously described in $\mathrm{AI}$.

There was no indication of a systematic map of sound space in AI. Instead, the mediolateral axis of AI contained spatially segregated regions of different unit classes. This organization was consistent with the previously described pattern of binaural interaction bands. The location-selective and location-insensitive unit classes might represent stages of fundamentally different lines of processing that are segregated within AI.
\end{abstract}

The primary auditory cortex (AI) of the cat contains a topographical representation of the auditory sensory

\footnotetext{
${ }^{1}$ The recording phase of these experiments was carried out in the laboratory of Dr. Masakazu Konishi at the California Institute of Technology. We are indebted to Dr. Konishi for his hospitality and advice throughout the course of this study. Dr. Eric I. Knudsen also was very helpful. The "hoop" was a product of the mechanical ingenuity of Herb Adams and the assistance of the Spencer Foundation. The manuscript benefitted from the critical reading by Drs. W. M. Jenkins, E. I. Knudsen, M. M. Merzenich, and J. M. Zook. This project was supported by a grant from the West Coast Neuroscience Consortium (to J. C. M.), National Institutes of Health-United States Public Health Service Grants EY 1909 and EY 1391 (to J. D. P.) and Grant NS-10414 (to Dr. M. M. Merzenich).

"Current address: National Vision Research Institute, 386 Cardigan St., Carlton 3053 Victoria, Australia.
}

epithelium, the cochlear partition (Merzenich et al., 1975). This is manifest as a caudal to rostral map of the audible frequency domain (tonotopic organization). In the visual system, the representation of the two dimensions of the retina fills the two dimensions of the visual cortex (e.g., Hubel and Wiesel, 1962). In contrast, the representation in the auditory cortex of the one-dimensional sound frequency domain is expanded laterally so that points on the cochlea (i.e., single frequencies) are represented as mediolaterally oriented lines in AI (isofrequency contours). This re-representation of single frequencies as isofrequency contours could provide a substrate for the topographical representation of some parameter of sound stimuli that is independent of sound frequency (e.g., Tunturi, 1952; Suga, 1977).

The response to binaural stimulation is one parameter that varies with location within an isofrequency contour. 
In $\mathrm{AI}$ of the cat, neurons with similar responses to binaural stimulation form radial columns extending through the depth of the cortex (Abeles and Goldstein, 1970; Imig and Adrian, 1977). Units with different responses to binaural stimulation are topographically segregated within $\mathrm{AI}$, forming rostrocaudally oriented bands ("binaural interaction bands") that cross the axis of isofrequency contours (Imig and Brugge, 1978; Middlebrooks et al., 1980). It has been suggested that bands of units that are sensitive to different features of dichotic stimuli might form independent processing units within AI (e.g., Middlebrooks et al., 1980). Such a topographical segregation of functional subunits has been demonstrated in the auditory cortex of the mustache bat, where neurons whose responses are suitable for the detection of low intensity sounds are segregated from neurons that could be useful for sound localization (Manabe et al., 1978).

The auditory cortex of the cat has been implicated in the representation of the spatial locations of sounds. For example, single units in AI of the cat tested with dichotic stimuli (sounds delivered independently to the two ears) are sensitive to interaural disparities that provide spatial information which is not available from one ear alone (Hall and Goldstein, 1968; Brugge et al., 1969). Some neurons in the auditory cortex respond preferentially to sounds presented in limited regions of the sound field (Evans, 1968; Eisenman, 1974; Sovijärvi and Hyvärinen, 1974). Bilateral ablations of the temporal cortex cause deficits in the ability of a cat to go to the source of a sound (see Neff et al., 1975 for review).

The present study was undertaken to examine the role of sound location in the functional organization within AI. Can single units in AI of the cat encode the location of a sound source? Might the mediolateral dimension of AI be concerned with spatial features of sound stimuli? We have addressed these questions by recording from single units in AI while presenting tonal stimuli in a free sound field. The receptive fields of auditory neurons were mapped by plotting the boundaries of spatial regions within which stimuli elicited a given neural response. Tangential electrode penetrations were oriented mediolaterally, approximately parallel to isofrequency contours, to explore the possibility that the spatial sensitivity of units might vary along the dimension where binaural interactions previously have been shown to vary. Using simple criteria based on characteristics of sensitivity to sound location, we could distinguish three classes of units in AI, two of which had sharply defined spatial receptive fields. These unit types were topographically segregated within isofrequency contours. By comparing the location sensitivity of cortical neurons with the acoustical transfer function of the external ear, we can begin to relate this data to the existing body of data collected in experiments using dichotic stimulation. These results are relevant to the neural bases of auditory behavior in the cat as well as to the understanding of the functional principles of organization within the primary auditory cortex. Preliminary reports of this work have appeared elsewhere (Middlebrooks and Pettigrew, 1980a, b).

\section{Materials and Methods}

Animal preparation and single unit recording. We studied 21 young adult cats from an inbred colony of specific-pathogen-free tabbies. Among these inbred cats, we found relatively little variation in the cortical sulcal pattern or in the location of the primary auditory cortical field (AI) relative to the sulcal pattern. This is in contrast to the great variation seen among cats from random sources. In addition, the cats from this closed colony were relatively free from ear mites and other pathology of the external and middle ear that is common among other cats. The neurophysiological data presented here are from 15 cats in which good recordings were obtained from AI. The data on the passive acoustics of the ear are from two additional cats.

Surgical anesthesia was induced with intramuscular injections of ketamine hydrochloride (Ketaset, $20 \mathrm{mg}$ / $\mathrm{kg}$ ) and diazepam (Valium, $1 \mathrm{mg} / \mathrm{kg}$ ). Supplementary doses of ketamine were given as needed, with smaller doses necessary once the animal was comfortably suspended in its hammock for recording (see below). Under even the lightest conditions of anesthesia used, we observed no spontaneous movements of the pinnae nor were there any movements observed in response to sound stimuli.

Following induction of anesthesia, cats were positioned temporarily in a stereotaxic device using blunt ear and eye bars. This facilitated alignment of the animal in a standard coordinate system. A stainless steel bar was attached to the skull with screws and acrylic cement. This bar extended behind the cat's head and was used later for holding the head in position in the sound chamber. The skull was opened over the middle ectosylvian gyrus in the left hemisphere and a recording chamber was mounted over the skull opening. The scalp was closed around the chamber and supporting bar, and the pinnae were restored to a normal-appearing configuration. At this point, the cat was removed from the stereotaxic device. Its body was suspended in a canvas hammock and the head was supported, still in alignment with stereotaxic coordinates, by clamping the steel bar from behind the head. Thus, the animal was positioned in a well defined coordinate frame with no obstructions in the sound path to its ears. This entire setup was positioned in the sound chamber with the cat's Horsley-Clarke horizontal plane parallel to the floor and the interaural axis aligned with the axis of the sound stimulation apparatus (Fig. 1).

The activity of single units was recorded with glassinsulated tungsten microelectrodes (Levick, 1972). Neural activity was amplified, band-pass filtered, and displayed on an oscilloscope and an audio monitor. In some cases, spikes were isolated with an amplitude discriminator and the output of the discriminator led to an event counter. Electrodes were positioned using a Narashige micromanipulator and a stepping motor microdrive. After the dura mater was opened and the electrode was positioned over the surface of the cortex, the recording chamber was filled with agar (3\% in isotonic saline) and covered with wax. This hydrostatically sealed chamber stabilized the brain and facilitated lengthy recordings from single cortical neurons. For most electrode penetrations, the electrode was oriented in the parasagittal plane and $10^{\circ}$ anterior to the frontal plane (see Fig. 7). In this orientation, the electrode passed approximately parallel to the isofrequency contours. In a few penetrations, the 

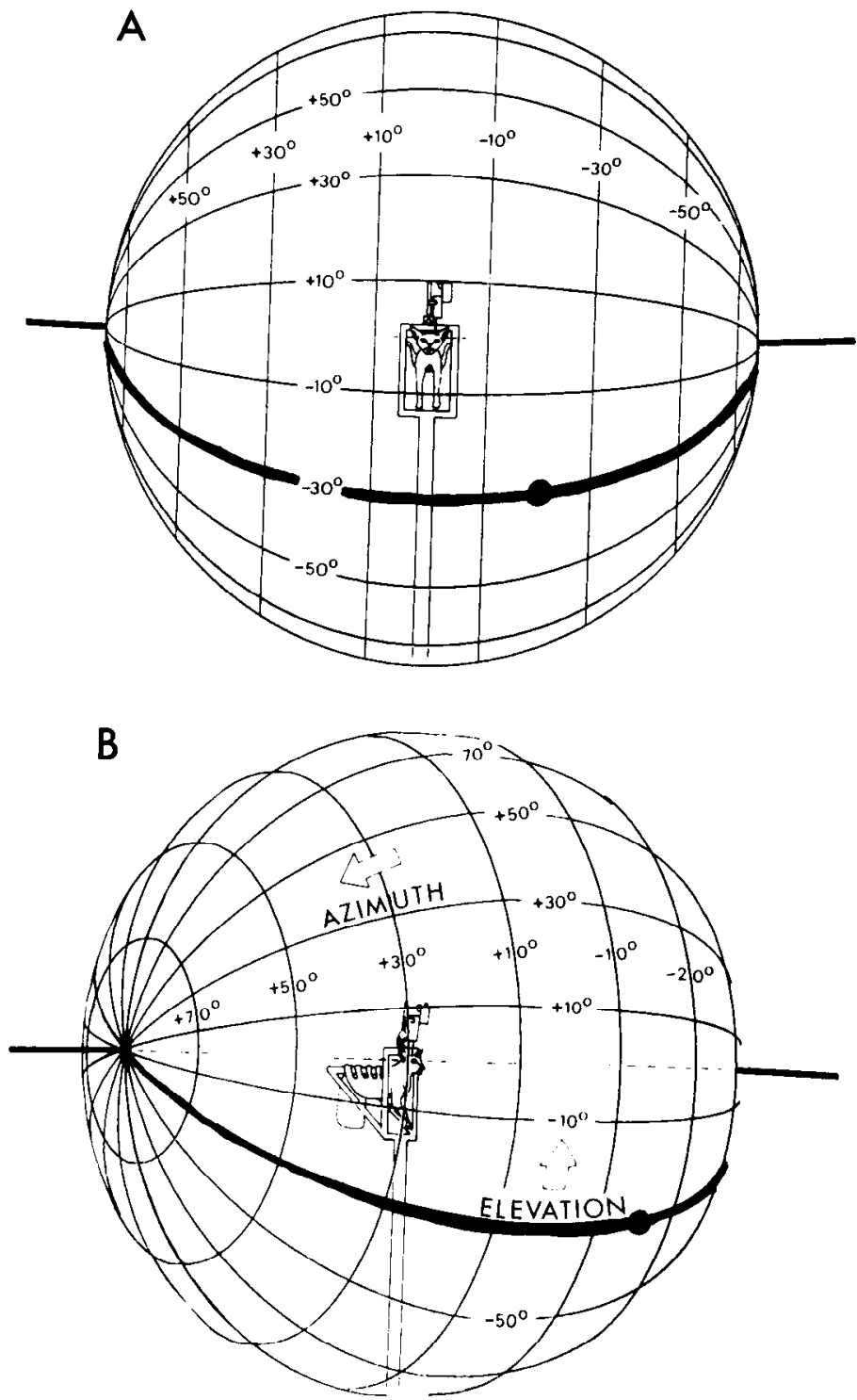

Figure 1. Position of cat within coordinate system. Use of this projection is consistent with the directions of motion of the loudspeaker on the hoop. Lines of constant elevation take the shape of the hoop. Movements of the loudspeaker in azimuth are accomplished by movements along a track on the hoop. Rotation of the entire hoop about its lateral poles causes movement of the loudspeaker in elevation with constant azimuth. The center of cat's interaural axis is concentric with the center of sphere. An elevation of $0^{\circ}$ is equivalent to the intersection of the Horsley-Clarke horizontal plane with the surface of the sphere; $0^{\circ}$ azimuth is on the midsagittal plane. The loudspeaker is shown positioned at $-20^{\circ}$ azimuth and $-30^{\circ}$ elevation. $A$, Coordinates were projected as if viewed from a point directly in front of cat at an infinite distance. $B$, The coordinate system was rotated $30^{\circ}$ about the vertical axis. The point directly in front of the cat $\left(0^{\circ}, 0^{\circ}\right)$ is shifted $30^{\circ}$ to the reader's right of center.

electrode was oriented approximately normal to the surface of the cortex, parallel to the axis of radial cell columns.

The location of the end of each electrode penetration was marked with an electrolytic lesion ( $10 \mu \mathrm{A}$ of cathodal current for $10 \mathrm{sec}$ ), and one or more additional lesions were made as the electrode was withdrawn. Following the recording period, the animal was deeply anesthetized with sodium pentobarbital, and isotonic saline followed by $10 \%$ formalin in isotonic saline was perfused through the heart. Electrode tracks were reconstructed from 50$\mu \mathrm{m}$ frozen sections stained with cresyl violet.

Sound stimulation. Sound stimuli were delivered using a system that moved a loudspeaker on the surface of an imaginary sphere, $1 \mathrm{~m}$ in radius, centered on the middle of the cat's interaural axis (Fig. 1). In this apparatus, the loudspeaker (Magnavox piezoelectric tweeter) moved along a semicircular track. This track (or "hoop") pivoted about an axis coinciding with the cat's interaural axis. We refer to movements along the track as movements in azimuth and to movements of the entire hoop about the interaural axis as movements in elevation. When the hoop was held at a constant elevation, movement of the speaker along the hoop described a meridian of iso-elevation. Conversely, when the speaker was fixed at a given azimuthal position on the hoop and the hoop was rotated about the axis passing through the interaural axis, the speaker described a parallel to the midsagittal plane (see Fig. 1). Thus, the coordinate system that we have used can be compared to a globe of the earth which has been rotated laterally so that the equator is in the midsagittal plane with parallels of latitude running vertically and the meridians of longitude running approximately horizontally. For simplicity we have used the terms azimuth and elevation in the text, but this unusual usage should be noted. The speaker could be positioned in any direction relative to the cat except for the area directly beneath the cat and the extreme $3^{\circ}$ of azimuth at the contralateral and ipsilateral poles. In practice, however, the positions of the loudspeaker in the sound hemifield in front of the cat were most intensively studied. The responses of cortical units to moving stimuli could not be studied conveniently as the responses to tonal stimuli were confounded by responses to the sounds produced by the mechanism moving the speaker.

The sound stimulation hoop and the animal were positioned inside a $5 \times 3 \times 3 \mathrm{~m}$ anechoic chamber (Industrial Acoustics Co.) rated at $300 \mathrm{~Hz}$. The controls for frequency, intensity, and location of sound stimuli were located outside of the sound chamber as were the recording oscilloscope and audio monitor.

Cats were stimulated with pure tone bursts that were $100 \mathrm{msec}$ in duration, presented one per second, with rise and fall times of $5 \mathrm{msec}$. The tones were generated with an audio oscillator (General Radio) and controlled in intensity with a decade attenuator (Hewlett-Packard). Characteristic frequencies (CFs) of units were determined by noting the frequency at which a given unit responded to the lowest stimulus intensity. Once the CF was determined for a given unit, the tone frequency was maintained constant while mapping that unit's spatial receptive field. Occasionally, responses to noise burst stimuli were observed and such stimuli were adequate to drive some units. However, no systematic study was made of the responses to noise, and all of the data presented in this report refer to responses to stimulation with tones of characteristic frequency.

Measurement of passive acoustics of head and pinnae. In two cases, the acoustic transfer function of the head and external ear was estimated by recording from the 
acoustic meatus with a probe microphone while presenting tones of constant frequency and varying the location of the sound source. The data plotted in Figures 8 and 9 are from an isolated cat head and neck. In this case, a small opening was made in the posterior aspect of the right pinna and a slit was cut in the cartilaginous portion of the acoustic meatus. A short length of polyethylene tubing was placed snugly over the end of a $1 / 8$-inch $B$ \& $\mathrm{K}$ microphone, extending approximately $5 \mathrm{~mm}$ beyond the diaphragm of the microphone. This probe fit tightly into the slit in the meatus and was cemented in place. The opening in the back of the pinna was closed so that the pinna regained its normal appearance. The cat head was suspended in the usual position in the sound chamber.

The output of the probe microphone was measured with a General Radio wave analyzer set to the $10-\mathrm{Hz}$ bandwidth. The AC output of the wave analyzer was read with a digital voltmeter. The sound pressure level in the acoustic meatus was measured while moving the piezoelectric loudspeaker systematically in azimuth and elevation in steps of $10^{\circ}$ or $15^{\circ}$. For each set of measurements, the frequency and intensity of the input to the loudspeaker were maintained constant and only the location was varied while the values on the voltmeter were recorded. For each frequency, intensities were expressed as decibels below the greatest level recorded. By expressing all the data as relative level for a constant frequency, we avoided errors that might have been introduced by slight deviations from a flat frequency response in our loudspeaker and recording configuration. By recording variations in sound level for a constant input to the loudspeaker, rather than adjusting the loudspeaker input to achieve a constant sound level, we place the requirement for linearity on the $\mathrm{B} \& \mathrm{~K}$ microphone rather than on the loudspeaker. In Figure 8, the solid circles indicate the locations of the loudspeaker for which intensity levels were recorded, and the curves (Figs. 8 and 9) are isointensity contours interpolated between the data points.

Graphic representation of data. In our sound stimulation apparatus, the loudspeaker moves on the surface of an imaginary sphere centered on the middle of the cat's interaural axis. The intersection of the cat's Horsley-Clarke horizontal plane with the sphere is defined as zero elevation, with positive elevations above this line. 'The intersection of the midsagittal plane (vertical plane) with the sphere is defined as zero azimuth, with azimuths on the cat's right side (the reader's left) defined as positive. Because all of the recordings in this study were made from the left cortical hemisphere, the sound hemifield containing positive azimuths is referred to as the contralateral hemifield. The straight line connecting the two lateral poles ( $\pm 90^{\circ}$ azimuth) passes through the cat's interaural axis.

In the figures in this report, the sphere surrounding the cat is flattened onto two dimensions as if viewed from an infinite distance. In some of the figures (e.g., Fig. 1A), the point of zero azimuth and zero elevation is drawn in the center of the figure. In this conformation, lines of constant azimuth (varying elevation) appear as straight, vertically oriented lines, and lines of constant elevation (varying azimuth) appear as arcs connecting the two lateral poles. Areas directly in front of the animal are most magnified, with more lateral regions appearing relatively compressed. In other figures (e.g., Fig. 1B), the coordinate system is rotated so as to bring the contralateral pole $30^{\circ}$ out of the plane of the paper. In this scheme, areas in the ipsilateral hemifield appear compressed relative to equivalent areas in the contralateral hemifield. The vertical midline $\left(0^{\circ}\right.$ azimuth) appears as an arc located $30^{\circ}$ to the reader's right of center. 'The advantage of this coordinate system is that more visual emphasis is placed on regions containing the majority of spatial receptive fields.

Receptive fields boundaries are indicated by solid circles located at every point at which the edge of a receptive field was measured. The lines connecting the solid circles are the shortest paths on the sphere between these points (i.e., great circles).

\section{Results}

Under ketamine anesthesia, we found the auditory cortex to be responsive and we were able to verify previously described features of its functional organization (e.g., Merzenich et al., 1975). Within the primary auditory field (AI), neurons were sharply tuned for frequency. Tonotopic organization was observed, with characteristic frequencies (CFs) increasing from the caudal to rostral pole of AI. These two properties, sharp tuning and correct gradient of $\mathrm{CF}$, were used as functional criteria to identify AI. The responses of most neurons in AI to pure tone bursts were transient, although neurons occasionally were encountered whose responses were sustained throughout the duration of the stimulus.

\section{Sensitivity to sound location}

Many neurons in AI were sensitive to the spatial location of a sound source. For those units, spatial receptive fields were plotted by mapping the region within which the presentation of a CF tone elicited a given neuronal response. Unless otherwise stated, receptive fields shown in the figures were plotted using a stimulus intensity $10 \mathrm{~dB}$ greater than the threshold found for the most effective location of the loudspeaker within the receptive field. The sound hemifields are identified as contralateral or ipsilateral relative to the cortical hemisphere containing the recording site.

All units encountered in this study could be grouped into one of three classes on the basis of their sensitivity to stimulus location. These classes were designated omnidirectional, hemifield, and axial units.

Omnidirectional units. Omnidirectional units responded to sounds presented from any direction in front of the cat. Often, a faintly delimited receptive field could be determined for these units at near-threshold intensities, but increases of the stimulus intensity to within 10 $\mathrm{dB}$ above threshold caused this "receptive field" to expand to fill the entire region explored in front of the animal. Although the response thresholds for a given omnidirectional unit were within $10 \mathrm{~dB}$ for all stimulus locations, the number of spikes elicited by a supra-threshold tone burst varied somewhat with sound location. For the majority of these units, the most effective stimuli were located in front of the animal near the vertical midline, with the threshold increasing gradually toward 
the lateral poles. Omnidirectional units were most readily distinguished from hemifield and axial units by their responses to stimuli located well into the ipsilateral sound hemifield.

Hemifield units. Hemifield units had receptive fields occupying most of the contralateral sound hemifield, with sharply defined vertically oriented medial borders (Fig. 2). The azimuth of the medial edge of the receptive field varied somewhat among hemifield units, although for most units it was within $20^{\circ}$ of the vertical midline. Laterally, the receptive field extended beyond the extreme contralateral pole of the sound field. These receptive fields sometimes had upper or lower boundaries, rarely both, but this was not a useful defining characteristic. The two receptive fields plotted in Figure 2 represent the extremes of variation within this class, one field having boundaries above and below as well as medially and the other having only a clear medial boundary.

Axial units. Axial units had completely circumscribed receptive fields located within the frontal contralateral quadrant of the sound field (Fig. 3). The location of these receptive fields coincided with the acoustical axis of the contralateral pinna (the passive acoustics of the pinna are described in a following section). A striking feature of the axial units was that the lateral edge of the receptive field terminated with a border which was as sharply defined as the medial border. These units were readily distinguished from hemifield units by failing to respond to stimuli presented at the contralateral pole of the sound field regardless of their intensity. Axial receptive fields had both upper and lower boundaries. The width of these receptive fields varied in azimuth from $20^{\circ}$ to $60^{\circ}$.

The locations of axial receptive fields varied somewhat throughout the entire course of these experiments, but within a given cat, the locations were remarkably constant (Fig. $4 A$ ). This can be seen in Figure $4 B$, where plotted symbols representing the centers of axial receptive fields are clustered for a given cat, while the locations of the clusters vary from cat to cat. The variation in axial receptive field locations between animals is most likely due to slight variations in the resting position of the pinnae under anesthesia and following the surgery to expose the cortex. The dependence of axial receptive field location on the position of the pinna was tested in one case by mapping the receptive field of an axial unit before and after moving the contralateral pinna with a clamp. Pulling the pinna medially had the expected effect of moving the receptive field medially (Fig. 5).

The boundaries of hemifield and axial receptive fields expanded somewhat with increasing stimulus intensity, although the amount of such expansion varied considerably among the units studied. Figure 6 shows examples of the extremes of this variation. For the hemifield unit whose receptive field is shown in Figure $6 A$, an increase in the stimulus intensity from $10 \mathrm{~dB}$ above threshold to $20 \mathrm{~dB}$ above threshold caused the medial edge of the
GZ-18 900B

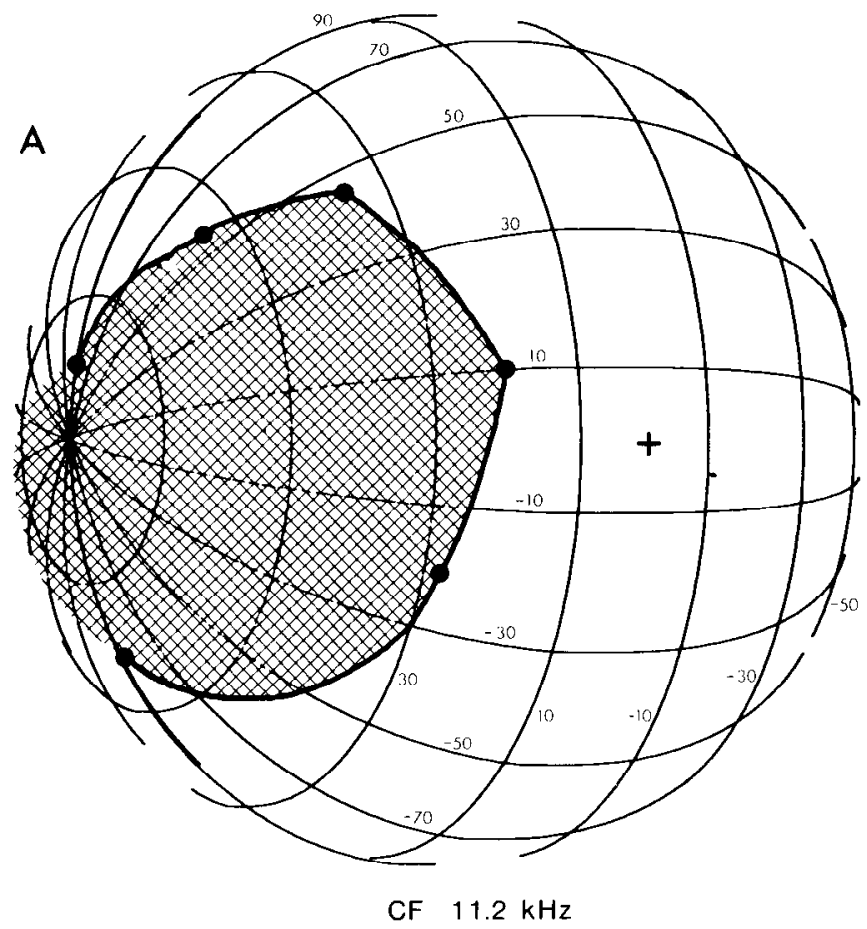

GZ $16490 D$

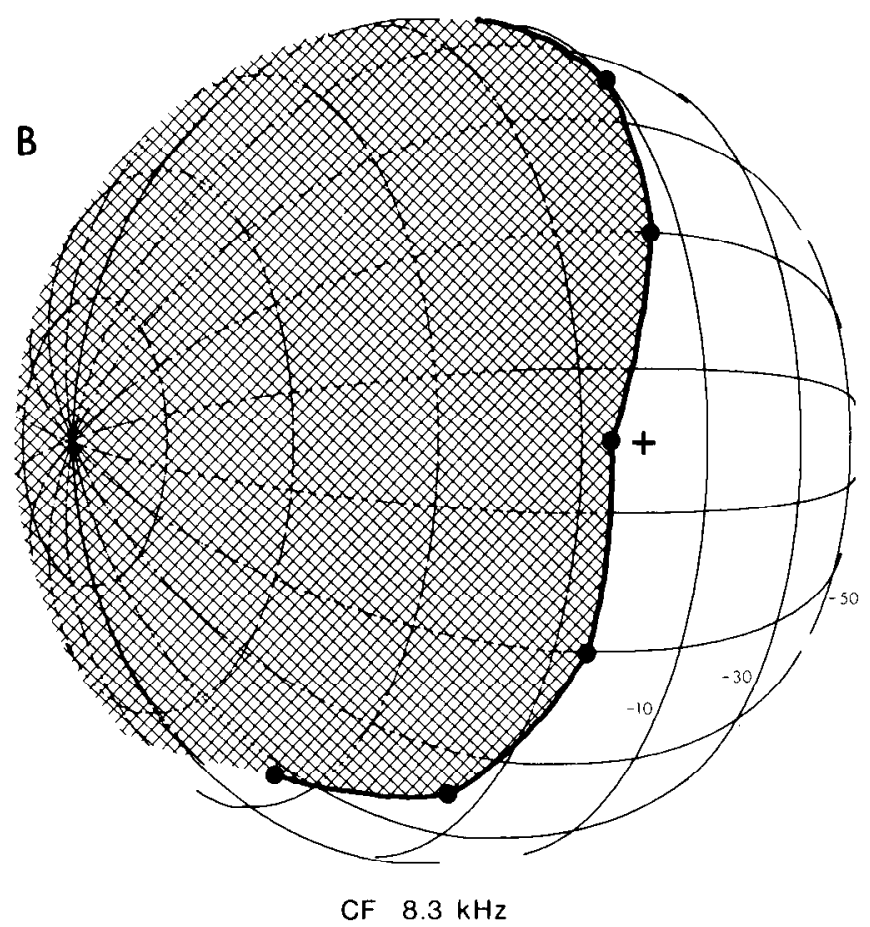

Figure 2. Two hemifield units. These two units represent the extremes of variation within this class. The cross indicates $0^{\circ}$ azimuth, $0^{\circ}$ elevation. Solid circles indicate locations where the borders of the receptive fields were determined. The lines connecting the solid circles are the shortest possible paths on the surface of the sphere (great circles). A, The receptive field of unit $900 \mathrm{~B}$ in cat GZ-18 has clear upper and lower borders as well as a medial border and extends beyond the contralateral pole. It was plotted with a characteristic frequency (CF) tone of $11.2 \mathrm{kHz}$. $B$, This receptive field (unit 490D, cat GZ-16) extended up as far over cat's head as was explored. The medial border extends slightly beyond midline, then sweeps down to make a partial lower border. CF $8.3 \mathrm{kHz}$. 


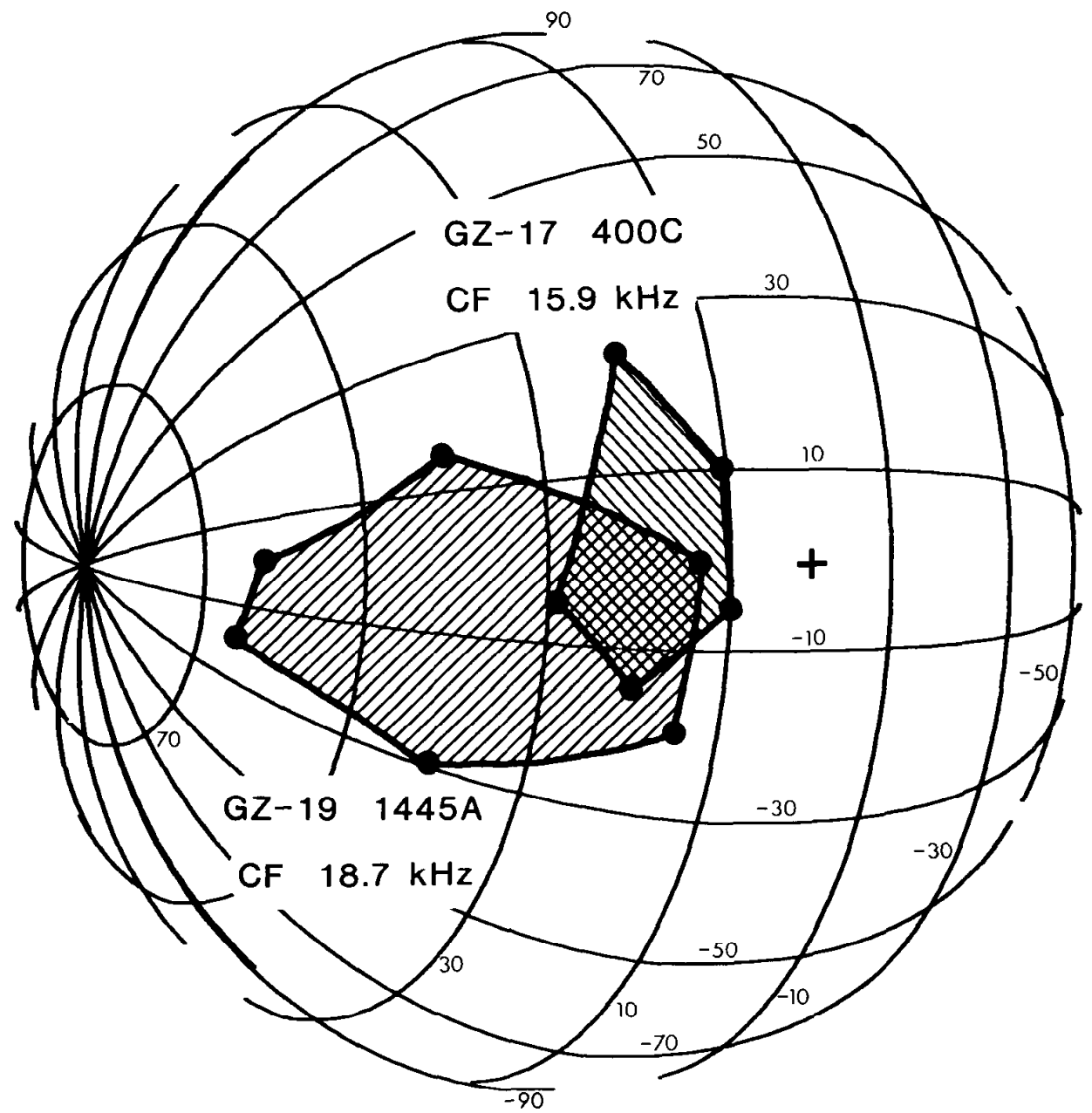

Figure 3. Two axial receptive fields. These two fields represent the extremes of variation within this class. The receptive field of unit $400 \mathrm{C}$ in cat GZ-17 is only $20^{\circ}$ wide in azimuth at its widest point and is elongated in elevation. The receptive field of unit $1445 \mathrm{~A}$ in cat GZ-19 is centered more laterally and is elongated in azimuth. Note that these two units were recorded in two different cats; within a single cat, axial receptive fields have roughly the same location (e.g., Fig. 4A).
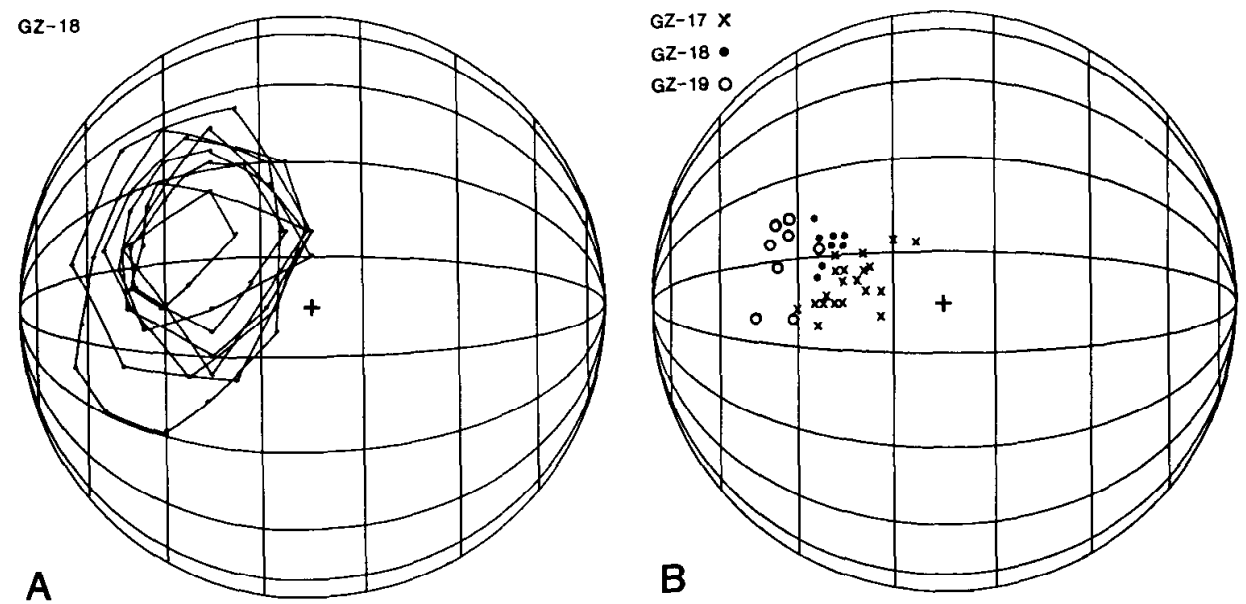

Figure 4. Locations of axial receptive fields. $A$, Boundaries of every axial receptive field encountered along one tangential penetration in cat GZ-18. CFs of these units ranged from 15.7 to $16.1 \mathrm{kHz}$. The receptive fields are centered approximately on the acoustical axis of the contralateral pinna (see Fig. 8). B, Every axial receptive field encountered in each of three cats. Symbols indicate the centers of receptive fields, with a different symbol for each cat. Cat GZ-17: 20 axial units recorded along $900 \mu \mathrm{m}$ of two electrode penetrations; CFs ranged from 15.5 to $16.9 \mathrm{kHz}$. Cat GZ-18: 8 units; $500 \mu \mathrm{m}$; one penetration; CFs from 15.7 to 16.1 $\mathrm{kHz}$. Cat GZ-19: 8 units; $1200 \mu \mathrm{m}$; one penetration; CFs from 14.2 to $19.1 \mathrm{kHz}$. 


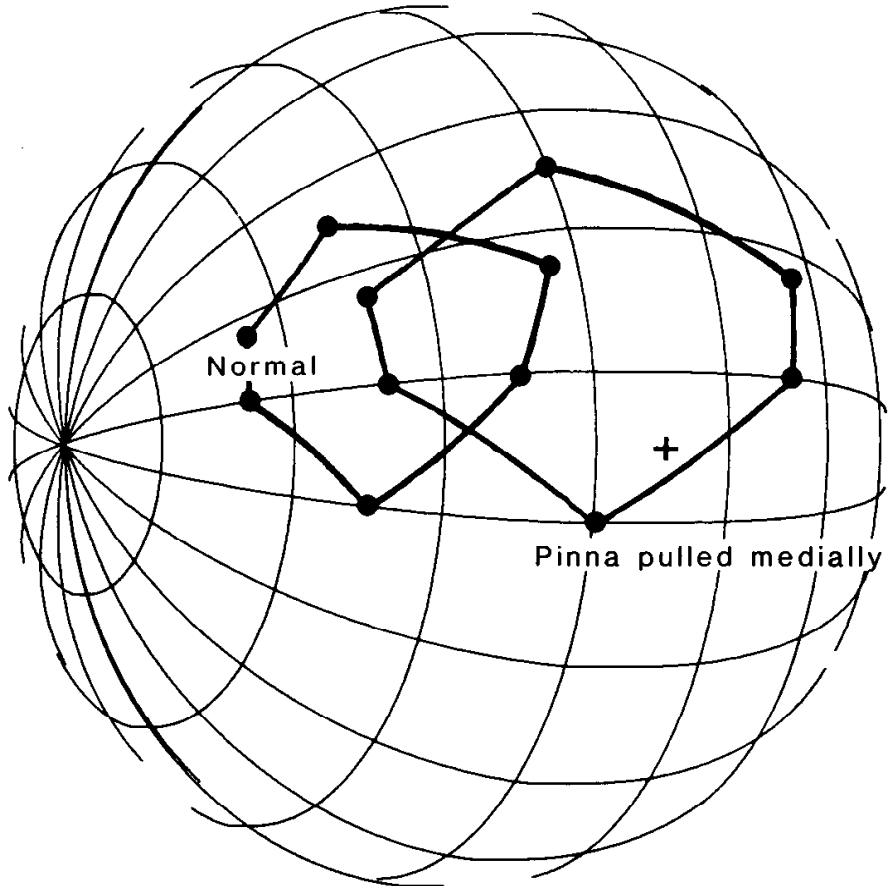

Figure 5. Dependence of axial receptive field location on pinna direction. Boundaries of axial receptive fields, both for the same unit, before and after the contralateral pinna was pulled medially with a clamp. Moving the pinna medially caused the receptive field to broaden slightly and move medially. Note that the variation in receptive field location is greater between cats than within a single cat. $\mathrm{CF} 18.5 \mathrm{kHz}$.

receptive field to move medially approximately $10^{\circ}$. In contrast, the medial borders of the receptive fields for 10 , 20 , and $30 \mathrm{~dB}$ above threshold for the unit shown in Figure $6 B$ are virtually identical. Likewise, the axial receptive field shown in Figure $6 \mathrm{C}$ more than doubled in diameter following a $15-\mathrm{dB}$ increase in stimulus intensity. Over a similar intensity range, the borders of the axial receptive field shown in Figure $6 D$ remained nearly unchanged.

\section{Distribution of response classes within AI}

Units with different types of receptive fields were spatially segregated within AI. Most electrode penetrations in this study were oriented oblique to the axis of radial cell columns and approximately parallel to isofrequency contours. In these penetrations, sequences of units displaying one type of spatial receptive field alternated with sequences of units with a different spatial receptive field type. Individual units with different receptive field properties tended not to intermingle along these penetrations. This is shown in Figure 7 where a tangential electrode penetration has been reconstructed. The CFs of units varied little along this penetration, indicating that the penetration was oriented along the axis of the isofrequency contours. There was, however, variation of the units' sensitivity to sound location as a function of their mediolateral position along the penetration. Omnidirectional, hemifield, and axial units clearly were separated into clusters along this 5-mm track. Along each of the few radial electrode penetrations which we made, all units encountered tended to belong to the same locationsensitive class.
Sequences of units of a given receptive field class sometimes were encountered more than once along a single tangential electrode penetration. This is demonstrated in the penetration reconstructed in Figure 7, where hemifield units were found in three discrete segments of a penetration, with the segments of hemifield units demarcated by sequences of omnidirectional or axial units. In each of four tangential penetrations that were oriented approximately parallel to isofrequency contours, two or more sequences of hemifield units were encountered, separated by 300 to $1000 \mu \mathrm{m}$ in which omnidirectional or axial units were recorded. In each of three other tangential penetrations, two segments of axial units were separated by 350 to $1250 \mu \mathrm{m}$ of recordings of omnidirectional or hemifield units.

\section{Relative sizes of unit populations}

In tangential electrode penetrations, the percentage of each penetration in which units of a given receptive field class were recorded should be proportional to the area of AI occupied by units of that class. In the nine cats in which units were recorded for more than $900 \mu \mathrm{m}$ of a tangential penetration (12 penetrations, all oriented approximately parallel to isofrequency contours), $52 \%$ of the total length was occupied by omnidirectional units, $23 \%$ by hemifield units, and $25 \%$ by axial units. These values should be taken only as a rough estimate of the

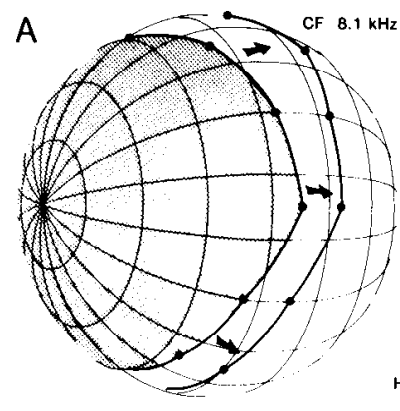

Hemifield Units
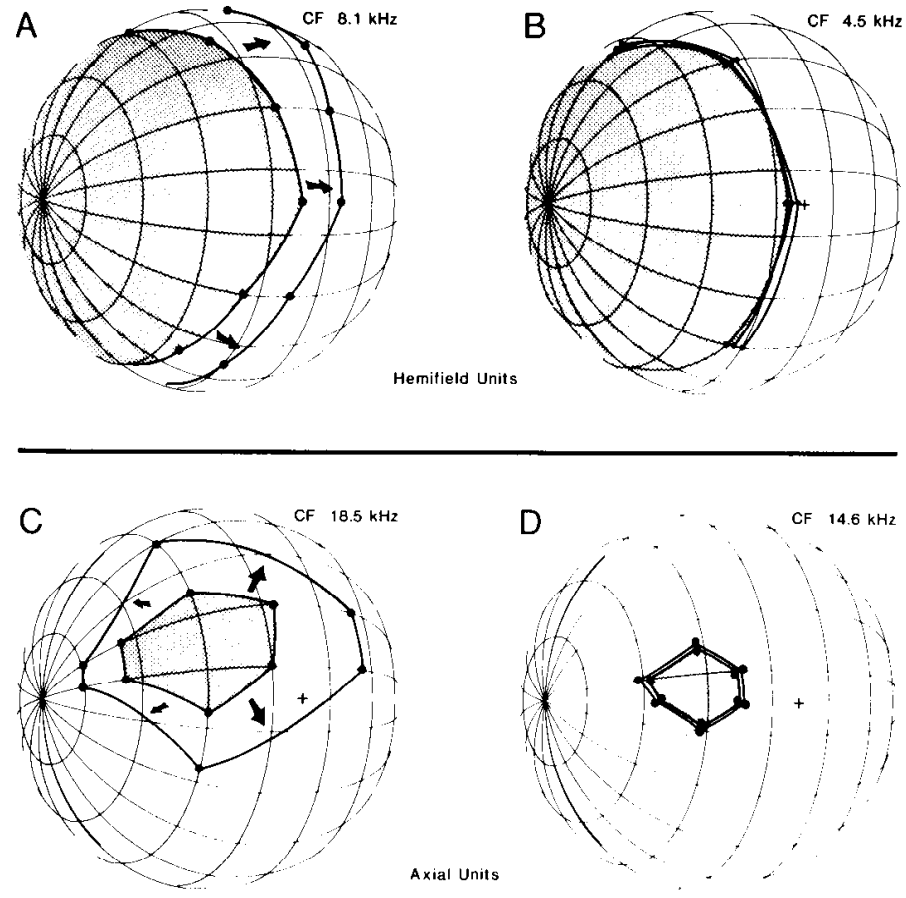

Figure 6. Expansion of receptive fields with increasing intensity. $A$, The hatched area is the receptive field of a hemifield unit plotted with a CF stimulus of intensity $10 \mathrm{~dB}$ greater than the threshold at the most sensitive loudspeaker location. $A r$ rows indicate the medial border of the receptive field of the same unit plotted with intensity $20 \mathrm{~dB}$ over threshold. $B$, Another hemifield unit. Three medial borders were plotted for intensities of 10,20 , and $30 \mathrm{~dB}$ above threshold. $C$, Axial unit. The hatched area is receptive field for $10 \mathrm{~dB}$ over threshold. Arrows show expansion with increase in intensity to $25 \mathrm{~dB}$ over threshold. $D$, Two receptive fields plotted for an axial unit with intensities of 10 and $25 \mathrm{~dB}$ over threshold. 


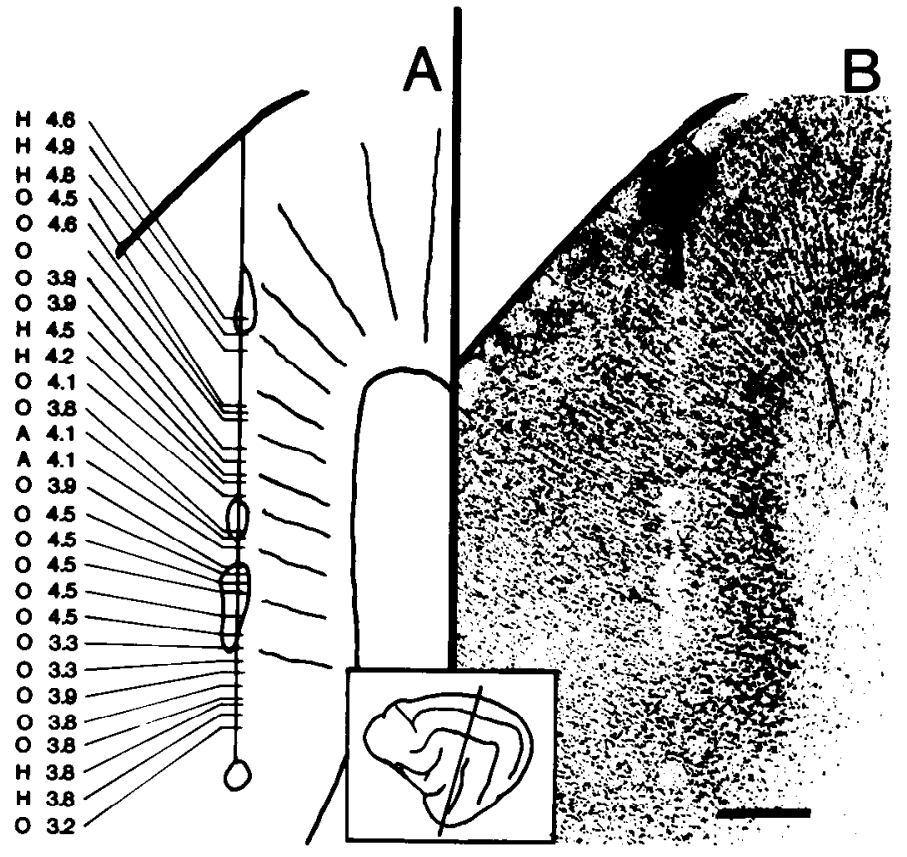

Figure 7. Reconstruction of tangential penetration. A, Numbers indicate $\mathrm{CF}$ in kilohertz. Letters indicate receptive field class: $O$, omnidirectional; $H$, hemifield; $A$, axial. Oblique lines suggest the orientation of radial cell columns. Irregular circles along track indicate approximate extent of marking lesions which were placed as the electrode was withdrawn. Note the segregation of different receptive field classes along this mediolaterally oriented penetration. $B$, Nissl-stained frontal section containing the electrode track drawn in $A$. Scale $=1 \mathrm{~mm}$. Inset, Lateral view of cat brain with the straight line indicating the orientation of the plane of section.

relative areas occupied by each unit class, as few of the penetrations traversed the entire mediolateral extent of AI, i.e., the medial and lateral extremes of the field were undersampled. This estimate of the relative area of AI devoted to each unit class is averaged over the entire portion of the rostrucaudal axis sampled (i.e., CFs from 3 to $20 \mathrm{kHz}$ ).

When the sampled population is divided into two groups on the basis of characteristic frequency greater than or less than $12 \mathrm{kHz}$, proportions are skewed heavily toward more hemifield units with lower CFs and more axial units with higher CFs. We chose $12 \mathrm{kHz}$ as the cutoff point for $\mathrm{CF}$ in the analysis because that frequency divides our data approximately in half and because no tangential penetrations crossed a $12-\mathrm{kHz}$ isofrequency contour. The ratios are 56:42:2 for omnidirectional:hemifield:axial units with CFs below $12 \mathrm{kHz}$ (six penetrations in six cats; total of $9650 \mu \mathrm{m}$ of tangential penetrations) and 49:5:46 for units with CFs above $12 \mathrm{kHz}$ (six penetrations in four cats; total of $10,000 \mu \mathrm{m}$ ). We lack the very large amount of data necessary to report the relative frequencies of occurrence of each unit class within more limited frequency bands. However, a striking feature is revealed by comparing the half of the sampled population having the lowest CFs with the half having the highest CFs. While the fraction of the population occupied by omnidirectional units is approximately independent of $\mathrm{CF}$, the fraction occupied by hemifield units at low fre- quencies is taken over by axial units at higher frequencies.

We cannot exclude the possibility that axial and hemifield receptive fields represent two ends of a continuum, with the lateral border of the axial receptive field gradually appearing as CF increases through the range around $12 \mathrm{kHz}$. However, spatial tuning does not seem to be strictly correlated with $\mathrm{CF}$, as the axial receptive fields encountered in one tangential penetration (Figure 4A) varied in diameter by a factor of 3 even though the CFs were restricted to a range of 15.7 to $16.1 \mathrm{kHz}$.

\section{Passive acoustics of the head and pinna}

The passive acoustical properties of the head and external ear must have a prominent effect on the spatial sensitivity of auditory neurons. This acoustical transfer function was estimated by measuring the sound pressure level inside the acoustic meatus with a probe microphone while presenting pure tones in the free sound field. The location of the loudspeaker was varied systematically while holding the tone frequency constant. For each frequency, data were plotted as decibels below the greatest level recorded, i.e., the datum for each point represents an attenuation relative to the level recorded for the most sensitive "axis" of the pinna. Iso-intensity contours are interpolated between the loudspeaker locations at which intensity levels were recorded.

The direction sensitivity of the right pinna at $4,8,16$, and $20.5 \mathrm{kHz}$ is shown in Figure 8 . The greatest ratio of maximum to minimum intensity levels measured for 4 $\mathrm{kHz}$ was $15 \mathrm{~dB}, 32 \mathrm{~dB}$ for $8 \mathrm{kHz}, 38 \mathrm{~dB}$ for $16 \mathrm{kHz}$, and $40 \mathrm{~dB}$ for $20.5 \mathrm{kHz}$. The iso-intensity contours tend to form ovals with the major axis oriented from superior and medial to inferior and lateral. For the greatest levels (the most sensitive direction of the pinna), the contour lines form closed loops centered on approximately $10^{\circ}$ to $20^{\circ}$ azimuth and $5^{\circ}$ to $15^{\circ}$ elevation. These closed contours resemble the shape and location of axial receptive fields (e.g., Fig. 4A). With increases in frequency (decreases in sound wavelength), the sensitivity gradient of the pinna becomes steeper, yet the center of the innermost contour line, the pinna's acoustical "axis," remains approximately constant in location. The centers of these contours, particularly for $16 \mathrm{kHz}$, seem displaced medially like the most medial of the axial receptive fields (e.g., Fig. $4 B, \mathrm{GZ}-17$ ). The insertion of the probe microphone from behind the pinna may have inclined the pinna slightly medially causing this apparent displacement. Note, however, that the elevation of the acoustical axis is very similar to the elevation of the centers of axial receptive fields in all cats and that this elevation also is close to that of the visual axis of the cat's eye $\left(13^{\circ}\right.$; Bishop et al., 1962).

In Figure 9, the data for 4,8 , and $20.5 \mathrm{kHz}$ are replotted as interaural intensity difference (IID) versus location in the contralateral sound hemifield. This figure is derived from the data shown in Figure 8 by assuming that the two pinnae are symmetrical and subtracting the sound levels (expressed in decibels) for loudspeaker locations that are mirror symmetrical with respect to the midsagittal plane $\left(0^{\circ}\right.$ azimuth). Contours of constant IID are drawn. In those plots, the contours for zero IID, by 

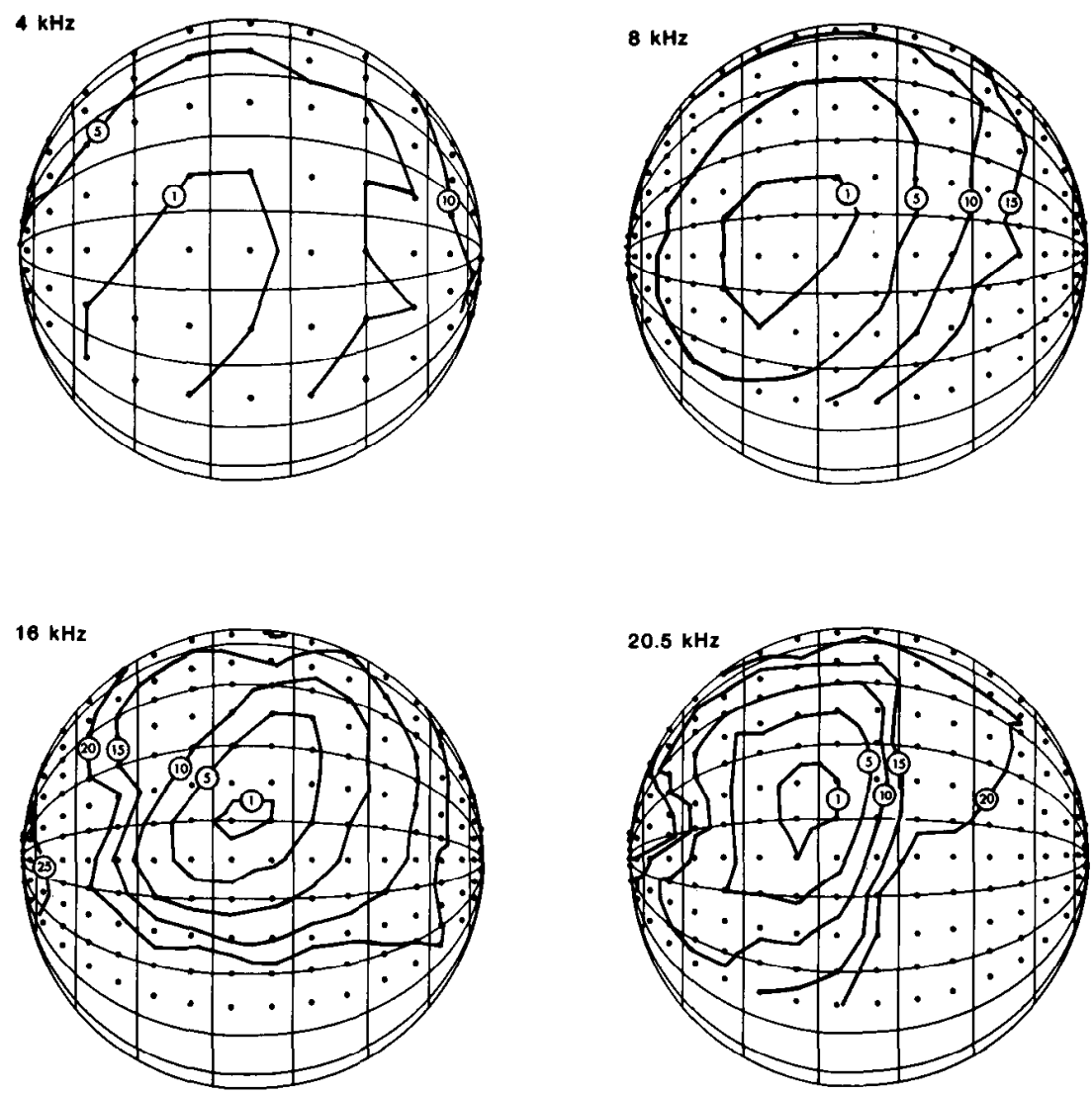

Figure 8. Directionality of the pinna. Intensity levels measured with probe microphone inside right acoustic meatus for $4,8,16$, and $20.5 \mathrm{kHz}$. Intensity levels are expressed as decibels below the greatest level recorded for each frequency. Solid circles indicate positions of the loudspeaker for which levels were measured. Curves are iso-intensity contours interpolated between data points. Numbers in circles indicate intensity level for each contour. The innermost closed contours encircle the acoustical axis centered at approximately $10^{\circ}$ to $20^{\circ}$ azimuth and $5^{\circ}$ to $15^{\circ}$ elevation.
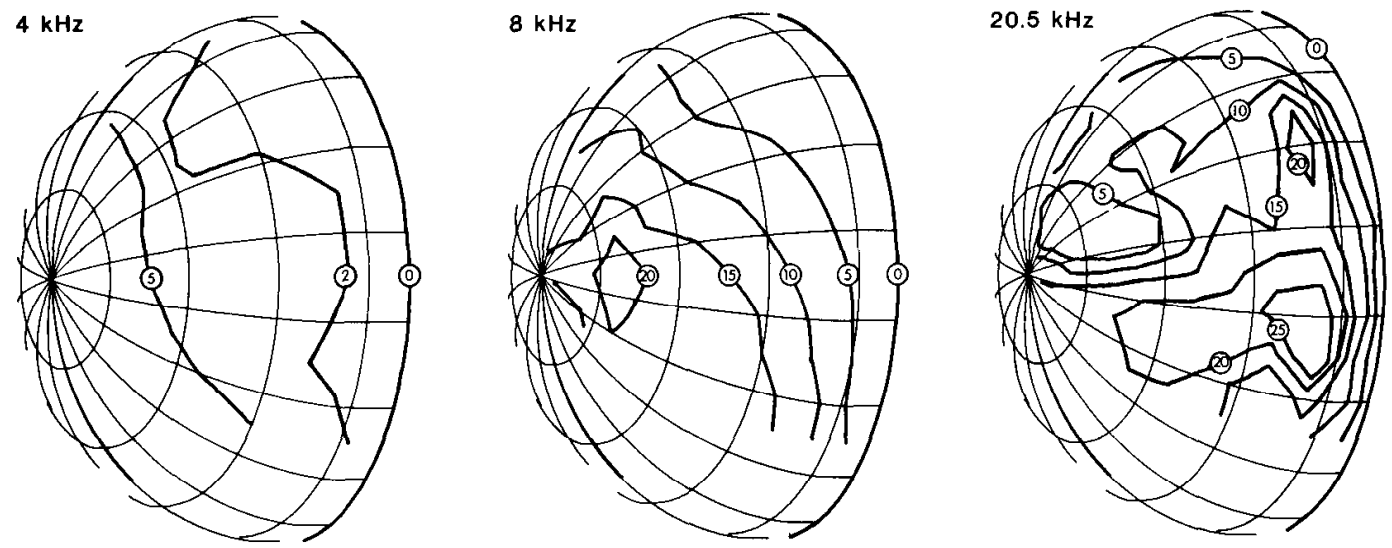

Figure 9. Interaural intensity differences for sounds located in the cat's right sound hemifield. Values are derived from Figure 8 assuming that the two ears are symmetrical with respect to midsagittal plane. Each level recorded for a loudspeaker position in the contralateral hemifield is divided by the level for a mirror-symmetrical position in the ipsilateral hemifield and each value is expressed in decibels. Curves are contours of constant IID. Numbers in circles indicate IID for each contour.

definition, fall on the midsagittal plane. In the plots for 4 and $8 \mathrm{kHz}$, the contour lines are oriented from superior and lateral to inferior and medial, with an even gradient of increasing IID oriented inferiorly and laterally. The plot for $20.5 \mathrm{kHz}$ is much more complicated, with reversals in the IID gradient and isolated IID maxima.

\section{Discussion}

The results of this series of experiments indicate that single units in the primary auditory cortex (AI) of the cat are sensitive to the location of sound stimuli. The characteristics of spatial receptive fields form a set of 
functional criteria by which every unit encountered in this study can be placed into one of three classes: (1) Omnidirectional units were relatively insensitive to sound locations. They responded to stimuli presented anywhere in the sound field, although the relative effectiveness of stimuli varied somewhat with sound location. These units made up $52 \%$ of the area of AI within the entire range of CFs sampled (approximately 3 to $20 \mathrm{kHz}$ ). (2) Hemifield units had receptive fields occupying all or most of the contralateral sound hemifield, extending beyond the extreme contralateral pole and with a well defined border near the vertical midline. These units occupied $42 \%$ of the population of units with CFs below $12 \mathrm{kHz}$, but only $5 \%$ of the population with CFs greater than $12 \mathrm{kHz}$. (3) Axial units had completely circumscribed receptive fields that encircled the acoustical axis of the contralateral pinna. The receptive field location was approximately constant for all such units within a single cat. Axial units occupied $46 \%$ of the population of units with CFs greater than $12 \mathrm{kHz}$ and only $2 \%$ of the population with CFs below $12 \mathrm{kHz}$. Units with different types of spatial sensitivity were segregated from each other within the cortex. Iso-intensity contours derived from physical measurements of the directionality of the ear form closed curves with some spatial features in common with the observed spatial receptive fields.

\section{Comparison with previous reports of location sensitivity}

Several previous reports have indicated that auditory cortical neurons are sensitive to sound location. Evans (1968) reported that approximately half of the units encountered in cat auditory cortex that were tested with "transient sound complexes" (snapping of fingers) gave preferential responses for particular stimulus locations. Some units responded only to stimuli presented in the contralateral hemifield, displaying a sharp diminution in their responses as the stimulus was moved a few degrees into the ipsilateral sound field.

Eisenman (1974) tested the responses of units in the auditory cortex of the cal to the presentation of tone and noise bursts. He found that for many units, plots of the number of spikes elicited by stimuli as a function of the azimuth of the source showed sensitivity to sound location. Notably, 33\% of 43 units studied responded more strongly to contralateral stimuli, and $16 \%$ displayed a peak response near $0^{\circ}$ azimuth. There were no spatial response profiles comparable to our axial receptive fields, although this might be expected since the separation of the loudspeaker positions in the previous study was larger than the size of many of the axial receptive fields.

Sovijärvi and Hyvärinen (1974) studied the responses of units in cat auditory cortex to continuous tonal stimuli presented from a hand-held loudspeaker. They found some units that responded only to stimuli moving in a particular direction in space. No indication was given in this report of the extent or location of regions from which stimuli were effective. In the current study, we found that most cortical units responded reliably only to the onset of our 100-msec tone bursts. The onset of a stationary tone burst, which was an adequate stimulus in our experiments, might be equivalent to the effect of moving a continuous tone source past a receptive field as in the study by Sovijärvi and Hyvärinen.
In the three earlier studies cited above, the location of AI was defined by cortical surface landmarks or by cytoarchitecture. Such anatomical criteria have since been demonstrated to be unreliable in locating the single cortical representation of the cochlea defined functionally (Merzenich et al., 1975). Data from a unit were included in the present study only if its response met the dual criteria of sharp tuning for frequency and correct position in the gradient of CFs increasing from caudal to rostral. Thus, some of the responses reported in earlier studies may not be directly comparable to those recorded in our experiments, as different criteria were used for defining "AI."

\section{Absence of a space map in $A I$}

Microelectrode recordings from the forebrain auditory structure, field L, of the owl have revealed the presence of single neurons with small, sharply delimited spatial receptive fields (Knudsen et al., 1977). In an auditory structure of the owl's midbrain, the MLD, units with small spatial receptive fields form a neural map of sound space, with an orderly shift in receptive field locations accompanying a shift in the location of the recording site within the nucleus (Knudsen and Konishi, 1978). No comparable map of sound space was observed in our recordings from AI of the cat. The most spatially restricted receptive fields, those of the axial units, were found in a location in the sound field that was constant for each cat. There was some variation in the location of the medial border of hemifield receptive fields, but we noted no orderly progression of these borders associated with advances of the recording electrode. Furthermore, the medial border of nearly all hemifield receptive fields fell within $20^{\circ}$ of the vertical midline.

\section{Directionality of the ear}

Previous studies of the passive acoustics of the external ear have concentrated on directionality in the horizontal plane. In one such study, Wiener et al. (1966) measured the sound pressure level within the cat's acoustic meatus as a function of tone frequency at each of five azimuths $\left( \pm 90^{\circ}, \pm 45^{\circ}\right.$, and $\left.0^{\circ}\right)$, all in the horizontal plane. For all frequencies, the greatest level was recorded when the sound was presented $45^{\circ}$ from the vertical midline. This is consistent with our observation that the center of isointensity contours for the directionality of the pinna, the acoustical "axis," remained approximately constant for all frequencies tested.

It is interesting to compare the acoustics of the cat's pinna with that of the human pinna. Shaw (1965) plotted the sound pressure recorded in the human acoustic meatus as a function of frequency for five different angles in the horizontal plane $\left( \pm 90^{\circ}, \pm 45^{\circ}\right.$, and $\left.0^{\circ}\right)$. The sound source producing the greatest level in the meatus varied between $+45^{\circ}$ and $+90^{\circ}$ as the sound frequency was changed. This variation in the orientation of the axis of the human pinna was substantially greater than that noted in our results and in those of Wiener et al. (1966) for the cat. Hebrank and Wright (1974) have shown that the elevation of the axis of the human pinna is even more dependent than azimuth on the frequency of a sound. Using artificial ears molded from human pinnae, they showed that the most sensitive elevation in the vertical 
midline plane changed from $60^{\circ}$ above horizontal to $30^{\circ}$ below horizontal with a change in sound frequency from 6 to $12 \mathrm{kHz}$ (measured from their Fig. 4). The differences between the pinnae of the cat and human could indicate a fundamental difference in the potential use of the external ear by the two species, perhaps also related to the fact that the behaving cat, but not man, is able to make both coordinated movements of both ears and isolated movements of each ear.

The frequency response profile of the human pinna contains narrow-band "notches" that move in the frequency domain as a function of sound source elevation (Hebrank and Wright, 1974). In our measurements of the directionality of the cat's pinna, sounds were varied systematically in the spatial domain for a limited number of frequencies. If such notches are present for the cat, they would appear as local minima in our directionality plots. No such pronounced minima were observed, although they might have been detected had we used a finer "grain" of loudspeaker placements.

\section{Derivation of spatial receptive fields from acoustical cues available to the nervous system}

The spatial receptive fields plotted for auditory cortical units resemble tactile and visual receptive fields determined for somatosensory and visual cortical units. However, the neural code for the location of acoustic stimuli is fundamentally different from the code for tactile and visual stimuli. Locations on the body surface or in the visual field are encoded directly as locations on the skin or retina, while spatial features of a sound source must be extracted within the central nervous system from multiple monaural and binaural stimulus cues. Among the most significant of these cues are interaural delay, interaural intensity differences (IIDs), spectral cues, monaural cues derived from the directionality of the pinna, and cues which rely on scanning movements of the head and pinnae.

Some of the above cues could not have contributed to the spatial sensitivity observed in the current study. (1) In our anesthetized preparation, no movements of the head were possible and no movements of the pinnae were observed in response to sounds. (2) Spectral cues could not have contributed to the sharpness of spatial receptive fields seen in the current experiments, as only pure tone stimuli were presented. We would not expect broad band spectral cues to contribute to the location sensitivity of neurons in AI, as single units in AI are sharply tuned for frequency (e.g., Merzenich et al., 1975). If there are cortical neurons that derive location information from a convergence of input from a broad region of the audible spectrum, they would be more likely to be found in one of the non-tonotopically organized cortical fields such as the second auditory field, AII, where single units have relatively flat frequency tuning curves (see Merzenich et al., 1979). (3) Interaural delay cues are a source of spatial information for low frequency sounds. However, in the existing report of delay sensitivity in the auditory cortex of the cat, no units with CFs greater than $2.5 \mathrm{kHz}$ were detected (Brugge et al., 1969), while most of the units sampled in the current study had CFs greater than 3 kHz.
Correlation of location sensitivity classes with classes defined with dichotic stimulation

The sensitivity of auditory cortical neurons in the cat to some binaural stimulus parameters has been tested in experiments in which sound stimuli were delivered independently to each ear (e.g., Hall and Goldstein, 1968; Brugge et al., 1969; Abeles and Goldstein, 1970). Many units in auditory cortex respond to monaural stimulation of either ear and their responses are facilitated by binaural in-phase stimulation. These units may be called "excitatory/excitatory" or "EE." Other units are excited by contralateral stimulation, but are inhibited by simultaneous ipsilateral stimulation ("excitatory/inhibitory" or "EI"). Some units respond maximally to binaural stimulus pairs containing an interaural delay ("delay sensitive" units; Brugge et al., 1969).

Omnidirectional units and EE units? The omnidirectional units observed in the current experiments were driven by stimuli presented anywhere in front of the cat with thresholds within $10 \mathrm{~dB}$ above the threshold for the most effective loudspeaker location. For all frequencies tested, the 10- $\mathrm{dB}$ contours for the directionality of the pinna nearly fill the portion of the sound field in front of the cat extending from the vertical midline to the lateral pole. A neuron receiving excitatory inputs from the contralateral and/or ipsilateral ears would be expected to have thresholds restricted to a range of $10 \mathrm{~dB}$ for stimuli presented anywhere in front of the cat. Thus, it seems likely that the omnidirectional units are equivalent to at least some of the EE neurons described previously.

Hemifield units and EI units? Hemifield units were driven by stimuli presented in the contralateral sound field, and the response ceased as the stimulus was moved into the ipsilateral field. Moving the sound source into the ipsilateral sound field is comparable to increasing the intensity of an ipsilateral stimulus in an experiment using dichotic stimulation. Thus, the hemifield units may be equivalent to EI neurons. In previous descriptions of EI neurons (e.g., Brugge et al., 1969; Middlebrooks et al., 1980 ), the greatest changes in the response rate of $\mathrm{EI}$ neurons occurred for contralateral and ipsilateral stimuli that were within $15 \mathrm{~dB}$ of equal intensity. This would correspond to a band of loudspeaker locations centered on the vertical midline, the region of the sound field containing the medial borders of most hemifield receptive fields. Brugge et al. (1969) demonstrated with dichotic stimulation that EI neurons in the cortex are most sensitive to changes in IID and are relatively insensitive to the net intensity of a binaural stimulus pair. Thus, one might expect the border of the receptive field of an EI unit to follow a contour of constant IID. The iso-IID contours derived in Figure 9 for 4 - and $8-\mathrm{kHz}$ tones resemble the medial borders of many of the hemifield receptive fields.

Axial units and EI units? The receptive fields of axial units have the shape and location of the innermost isointensity contours for the contralateral ear derived from physical measurements of pinna directionality. Most of the axial units had CFs greater than $12 \mathrm{kHz}$, the range of frequencies for which the pinna is most directional. Both the location of axial receptive fields and the location of the axis of the pinna are approximately constant within a single cal for a range of sound frequencies. When the 
contralateral pinna is manipulated by the investigator, axial receptive fields move in the same direction as the pinna. These points argue that the responses of axial units are dominated by excitatory inputs from the contralateral ear. However, the receptive field of such a monaural unit would be expected to expand greatly with increasing intensity, just as the iso-intensity contours in Figure 8 expand. In contrast, axial receptive fields expand with intensity no more than the field shown in Figure $6 C$. In experiments using dichotic stimulation, monaural units seldom are recorded in the cortex; most unit responses are either facilitated or inhibited by ipsilateral stimulation (Imig and Adrian, 1977; Middlebrooks et al., 1980). For three axial units studied in the current study, blocking the ipsilateral ear with a ball of cotton made the boundaries of the receptive fields more sensitive to increases in stimulus intensity. This suggests a role of ipsilateral inputs in "sharpening" the spatial tuning of axial units.

Could axial units correspond to EI units? In our protocol, locations of the loudspeaker near the axis of the contralateral pinna, i.e., within axial receptive fields, were the most comparable to a contralateral monaural stimulus, the most effective stimulus for an EI neuron. Movements of the loudspeaker away from this axis would decrease the ratio of contralateral to ipsilateral intensity, somewhat analogous to increasing the strength of the ipsilateral input in a dichotic stimulation experiment. Such a movement of the loudspeaker would place it outside of an axial unit's receptive field, much as increasing the intensity of an ipsilateral stimulus inhibits the response of an EI unit.

\section{Spatial receptive field properties and functional organization within AI}

Recent experiments using dichotic stimulation and microelectrode mapping techniques have revealed some basic features of functional organization in AI. Imig and Adrian (1977) demonstrated that neurons arrayed in radial columns through the depth of AI have similar responses to binaural (dichotic) stimulation (as suggested by Abeles and Goldstein, 19?0), and they showed that these binaural columns are elaborated for varying distances in the plane of the cortex. In each binaural column, all responses encountered were EE (excitatory/excitatory) or all responses were EI (excitatory/inhibitory). Over the range of CFs encountered (approximately 3 to $20 \mathrm{kHz}$ ), EE units occupied two-thirds and EI units occupied one-third of the sampled population. Middlebrooks et al. (1980) showed that the binaural columns form multiple radially organized bands oriented rostrocaudally across the axis of isofrequency contours and extending over the length of AI. The primary field in each hemisphere usually contains three EI bands separated by two EE bands. This banded organization also is demonstrable in the pattern of transcallosal connections between AI in the two hemispheres (Imig and Brugge, 1978). It was suggested from the microelectrode mapping data as well as from existing connectional and cytoarchitectonic evidence that the binaural interaction bands in AI might represent independent cortical processing units with fundamentally different roles in cortical function (Middlebrooks et al., 1980).
In the current experiments, units with different classes of sensitivity to sound location were segregated along both tangential and radial electrode penetrations. At lower frequencies (below $12 \mathrm{kHz}$ ), sequences of omnidirectional units alternated along tangential penetrations with sequences of hemifield units. For higher CFs, sequences of omnidirectional units alternated with sequences of axial units. Sequences of units of a given response class were encountered more than once along a single penetration. It was argued above that omnidirectional units probably are equivalent to $\mathrm{EE}$ units and that hemifield and axial units might be equivalent to EI units. Confirmatory experiments remain to be done, but the current evidence is most consistent with the interpretation that AI is subdivided by a pattern of bands of omnidirectional units alternating with bands of hemifield and axial units that is comparable to the pattern of alternating EE and EI bands shown previously.

\section{Comparison of AI with VI}

There are a number of similarities and contrasts to be pointed out between the functional organization just described for AI and the well characterized functional organization of the primary visual cortex, VI. For example, the functional segregation into receptive field classes which has been emphasized in the visual cortex is based on laminar differences in the incidence of neurons with "simple," "complex," and "end-stopped" receptive fields (Gilbert and Wiesel, 1979). In the present case, the functional segregation into omnidirectional, axial, and hemifield units is not based on a laminar subdivision of radial cell columns but rather upon a topographic subdivision of the mediolaterally oriented isofrequency contours, probably correlated with the system of binaural interaction bands. In this respect, a more appropriate comparison might be made with the functional subdivision of VI into ocular dominance bands which are superimposed on the cortical map of the retina.

Binaural interaction bands and ocular dominance bands both may reflect a substrate for the representation of information extracted by comparison of bilateral inputs: sound location from binaural interaction and visual depth relative to the fixation plane from binocular interaction (e.g., Cooper and Pettigrew, 1979). Although topographic segregation of binocular units with respect to target depth relative to the fixation plane has not been demonstrated in the visual cortex of the cat, such segregation has been observed in the visual cortex of ungulates (Clarke et al., 1976). This raises the possibility that the functional organization of AI and VI will prove ultimately to be more comparable than present data indicate.

\section{AI and sound localization behavior}

Ablation behavioral studies have implicated the auditory cortex in the localization of sound. Large bilateral lesions of the temporal cortex can almost completely disrupt the ability of a cat to localize a sound (e.g., Neff et al., 1956; Riss, 1959). Unilateral lesions cause pronounced behavioral deficits only in tests where cats must distinguish between the locations of transient sounds presented within the contralateral hemifield (Jenkins, 1980). It is difficult to compare the classical behavioral studies with the current study of single units in AI, as 
recent mapping studies have demonstrated that the location of AI is defined only approximately by the anatomical landmarks used for delimiting ablations. There has been no published report of a deficit in sound localization behavior in a cat following the placement of a lesion that was restricted to AI as defined by single unit or other evoked potential recording method.

Axial units have the most restricted receptive fields of all units recorded in AI. Yet, all of the axial units in AI of a given hemisphere appear to be trained on the same region of auditory space, presumably the region along the axis of the contralateral pinna. These units would be effective in localizing a sound only if the cat could make scanning movements of the head and pinna, while a normal cat can identify the source of a sound even if the stimulus is a click so brief that the cat has no time to scan (Jenkins, 1980). Alternatively, hemifield units would at least indicate the laterality of the stimulus. A click presented at the extreme lateral pole of the sound field would be expected to activate hemifield and omnidirectional units in the contralateral cortical hemisphere, but would activate only omnidirectional units in the ipsilateral hemisphere.

The two location-selective unit classes found in AI, axial and hemifield, were associated with different ranges of CF, with most axial units displaying CFs higher than the majority of hemifield units' CFs. Presumably, the only location-selective units that would be activated in AI by a high frequency tone would be the axial units, and likewise, the hemifield units would be the only location-selective units activated by lower frequency tones. In nature, a cat rarely encounters a pure tone stimulus, yet cats in a laboratory are able to discriminate the locations of pure tone stimuli separated by as little as $10^{\circ}$ to $20^{\circ}$ (however, this has been shown only when the stimuli were long enough to scan; Casseday and Neff, 1973). It is difficult (as well as unrealistic) to explain the available sound localization data entirely within the context of the location-selective units that we have encountered in AI. It would appear that other mechanisms must be at work in sound localization, perhaps in one or more of the other auditory cortical fields.

We favor a view of the axial units as part of a mechanism for a cat to scrutinize a sound source of interest. Cats can be observed exploring their environments with independent movements of both pinnae, effectively placing novel sounds into the receptive fields of their axial units. If a sound of particular interest is detected, the cat orients its head and both pinnae toward the source. This motion would devote the bands of axial units in both hemispheres to analysis of the sound, also helping to exclude extraneous sounds located more laterally. In this regard, it perhaps is significant that the axial receptive fields and the acoustical axes of the pinnae are aligned with the cat's visual axes (Bishop et al., 1962).

\section{Conclusion}

By systematically varying the locations of sound stimuli presented in a free sound field, we have revealed the presence in $\mathrm{AI}$ of units that are sensitive to sound location in two dimensions, azimuth and elevation. Yet, we also have raised the likelihood that AI is not the principal cortical substrate for an internal map of auditory space.
The omnidirectional units, the largest class encountered in these experiments, have little or no selectivity for sound location. The receptive fields of the two locationselective unit classes appear not to cover the contralateral sound field with resolution comparable to that observed in behavioral studies. However, the classification of units by spatial sensitivity does reveal a form of topographical organization within the mediolateral dimension of AI. The unit types distinguished in this way might represent stages of two fundamentally different lines of processing of auditory information that are topographically segregated in AI. One system, represented in AI by the omnidirectional units, appears to contribute to detection and/or analysis of nonspatial features of sounds. The other location-selective system comprises the hemifield units for lower frequencies and the axial units for higher frequencies. Activity in the hemifield units could signal the approximate location, left or right, of a sound. The axial units constitute a cortical region devoted to the scrutiny of sounds aligned with the axis of the external ear.

\section{References}

Abeles, M., and M. H. Goldstein, Jr. (1970) Functional architecture in cat primary auditory cortex. J. Neurophysiol. 33: 172-187.

Bishop, P. O., W. Kozak, and G. J. Vakkur (1962) Some quantitative aspects of the cat's eye: Axis and plane of reference, visual field coordinates, and optics. J. Physiol. (Lond.) 163: 466-502.

Brugge, J. F., N. A. Dubrovsky, L. M. Aitkin, and D. J. Anderson (1969) Sensitivity of single neurons in the auditory cortex of cat to binaural stimulation: Effects of varying interaural time and intensity. J. Neurophysiol. 32: 1005-1024.

Casseday, J. H., and W. D. Neff (1973) Localization of pure tones. J. Acoust. Soc. Am. 54: 365-372.

Clarke, P. G. H., I. M. L. Donaldson, and D. Whitteridge (1976) Binocular visual mechanisms in cortical areas I and II of the sheep. J. Physiol. (Lond.) 256: 509-526.

Cooper, M. L., and J. D. Pettigrew (1979) A neurophysiological determination of the vertical horopter in the cat and owl. J. Comp. Neurol. 184: 1-25.

Eisenman, J. M. (1974) Neural encoding of sound location: An electrophysiological study in auditory cortex (AI) of the cat using free field stimulation. Brain Res. 75: 203-214.

Evans, E. F. (1968) Cortical representation. In Hearing Mechanisms in Vertebrates, A. V.S. de Reuck and J. Knight, eds., pp. 272-287, Churchill, London.

Gilbert, C. D., and T. N. Wiesel (1979) Morphology and intracortical projections of functionally characterised neurones in the cat visual cortex. Nature 280: 120-125.

Hall, J. L., III, and M. H. Goldstein, Jr. (1968) Representation of binaural stimuli by single units in primary auditory cortex of unanesthetized cats. J. Acoust. Soc. Am. 43: 456-461.

Hebrank, J., and D. Wright (1974) Spectral cues used in the localization of sound sources on the medial plane. J. Acoust. Soc. Am. 56: 1829-1834.

Hubel, D. H., and T. N. Wiesel (1962) Receptive fields, binocular interaction, and functional architecture in the cat's visual cortex. J. Physiol. (Lond.) 160: 106-154.

Imig, T. J., and H. O. Adrian (1977) Binaural columns in the primary field (AI) of cat auditory cortex. Brain Res. 138: 241257.

Imig, T. J., and J. F. Brugge (1978) Sources and terminations of callosal axons related to binaural and frequency maps in primary auditory cortex of the cat. J. Comp. Neurol. 182: 637-660. 
Jenkins, W. M. (1980) Sound localization: Effects of unilateral lesions in the central auditory system. Doctoral dissertation, Florida State University.

Knudsen, E. I., and M. Konishi (1978) Space and frequency are represented separately in auditory midbrain of the owl. J. Neurophysiol. 41: 870-884.

Knudsen, E. I., M. Konishi, and J. D. Pettigrew (1977) Receptive fields of auditory neurons in the owl. Science 198: 12781280.

Levick, W. R. (1972) Another tungsten microelectrode. Med. Electron. Biol. Eng. 10: 510-513.

Manabe, T., N. Suga, and J. Ostwald (1978) Aural representation in the Doppler-shifted-CF processing area of the auditory cortex of the mustache bat. Science 200: 339-342.

Merzenich, M. M., P. L. Knight, and G. L. Roth (1975) Representation of the cochlea within the primary auditory cortex in the cat. J. Neurophysiol. 38: 231-249.

Merzenich, M. M., R. A. Andersen, and J. C. Middlebrooks (1979) Functional and topographic organization of the auditory cortex. Exp. Brain Res. 2 (Suppl): 61-75.

Middlebrooks, J. C., and J. D. Pettigrew (1980a) Functional classes of auditory cortical neurons distinguished by sensitivity to sound location. Soc. Neurosci. Abstr. 6: 333.

Middlebrooks, J. C., and J. D. Pettigrew (1980b) Spatial receptive fields of neurons in the primary auditory cortex (AI) of the cat. J. Acoust. Soc. Am. 68 (Suppl.): in press.

Middlebrooks, J. C., R. W. Dykes, and M. M. Merzenich (1980) Binaural response-specific bands in primary auditory cortex
(AI) of the cat: Topographical organization orthogonal to isofrequency contours. Brain Res. 181: 31-48.

Neff, W. D., J. F. Fisher, I. T. Diamond, and M. Yela (1956) Role of auditory cortex in discrimination requiring localization of sound in space. J. Neurophysiol. 19: 500-512.

Neff, W. D., I. T. Diamond, and J. H. Casseday (1975) Behavioral studies of auditory discrimination: Central nervous system. In Handbook of Sensory Physiology, W. D. Keidel and W. D. Neff, eds., Vol. 2, pp. 307-400, Springer, New York.

Riss, W. (1959) Effect of bilateral temporal cortical ablation on discrimination of sound direction. J. Neurophysiol. 22: 374384.

Shaw, E. A. G. (1965) Far canal pressure generated by a free sound field. J. Acoust. Soc. Am. 39: 465-470.

Sovijärvi, A. R. A., and J. Hyvärinen (1974) Auditory cortical neurons in the cat sensitive to the direction of sound source movement. Brain Res. 73: 455-471.

Suga, N. (1977) Amplitude spectrum representation in the Doppler-shifted-CF processing area of the auditory cortex of the mustache bat. Science 196: 64-67.

Tunturi, A. R. (1952) A difference in the representation of auditory signals from the left and right ears in the isofrequency contours of the right middle ectosylvian auditory cortex of the dog. Am. J. Physiol. 168: 712-727.

Wiener, F. M., R. R. Pfeiffer, and A. S. N. Backus (1966) On the sound pressure transformation by the head and auditory meatus of the cat. Acta Otolaryngol. (Stockh.) 61: 255-269. 